\title{
Astrometry from mutual event and small-separation CCD imaging $\dagger$
}

\author{
Q. Y. Peng ${ }^{1,3}$, N. V. Emelyanov ${ }^{2}$, L. Zhou ${ }^{1}$ and W. R. Gu ${ }^{1}$ \\ ${ }^{1}$ Department of Computer Science, Jinan University, Guangzhou 510632, China \\ email: pengqy@pub.guangzhou.gd.cn \\ ${ }^{2}$ Sternberg Astronomical Institute, 13, Universitetskij prospect, 119992 Moscow, Russia \\ email: emelia@sai.msu.ru \\ ${ }^{3}$ Joint Laboratory for optical astronomy, CAS, Kunming 650011, China
}

\begin{abstract}
In order to determine precisely positions of the Galilean satellites of Jupiter, it is useful that shortly before and/or after photometric observations of a mutual event, normal CCD imaging observation is also performed for the two small-separation satellites. Experimental observations showed that the two observational types, on the whole, could derive mean $(\mathrm{O}-\mathrm{C}) \mathrm{s}$ with very good internal and external agreement (about 15-20 mas).
\end{abstract}

Keywords. planets and satellites: general, astrometry, eclipses, occultations

\section{Introduction}

At present, the newest theories of the Galilean satellites are the L1 in the IMCCE and JUP230 in NASA/JPL. Among modern ground-based CCD observations, normal CCD imaging and mutual event are two commonly used types for precise measurement of the Galilean satellites. Normal CCD imaging refers to the measurement of relative coordinates in the focal plane of the used telescope, and mutual event to the measurement of the epoch of an event (an eclipse or occultation of a satellite due to another satellite). Normal CCD imaging needs usually a long focal length telescope but it is not easy to calibrate its small field of view of the CCD due to the shortage of the suitable reference stars. However, for a small-separation satellite-pair, the calibration could be good enough since we can deliver calibration parameters (scale factor and orientation) by comparing the pixel positions of greater-separation satellites with their positions from theoretical ephemerides. These parameters would have enough precision for the measurement of small-separation satellite-pair. Therefore, it is very useful that before and/or after photometric observation of a mutual event, normal CCD imaging is also performed for the two small-separation satellites. In this paper, we describe our two types of observations taken at the Yunnan Observatory by 1-m telescope and their reductions, and show their results.

\section{Observations and their reduction}

In 2003, some mutual events of the Galilean satellites of Jupiter predicted by Arlot (2002) were observed at the Yunnan Observatory by the 1-m telescope with $1024 \times 1024$ CCD. Seven effective light curves for the mutual events were obtained. For four of these mutual events, we also performed normal CCD imaging observations before these events. In order to measure the pixel position and photometry of the Galilean satellites, we used a

$\dagger$ supported by the National Science Foundation of China (Grant Nos 10573008 and 10778617) 
Table 1. (O-C) results (unit: arcsec)derived from mutual events and normal CCD imaging, respectively. J1, J2, J3 and J4 represent Io, Europa, Ganymede and Callisto, respectively.

\begin{tabular}{cccccc}
\hline Obs date & Event & Mutual & event & $\mathrm{CCD}$ & imaging \\
\hline & & $(\mathrm{O}-\mathrm{C}) \mathrm{x}$ & $(\mathrm{O}-\mathrm{C}) \mathrm{y}$ & $\langle(\mathrm{O}-\mathrm{C}) \mathrm{x}\rangle$ & $<(\mathrm{O}-\mathrm{C}) \mathrm{y}\rangle$ \\
\hline Feb 18 & J4OJ3 & -0.053 & 0.117 & 0.034 & 0.025 \\
Feb 20 & J1OJ2 & -0.014 & 0.021 & -0.028 & 0.028 \\
Feb 27 & J1OJ2 & -0.030 & 0.015 & -0.036 & 0.034 \\
Feb 28 & J1EJ4 & 0.052 & 0.176 & -0.012 & -0.004 \\
\hline
\end{tabular}

Notes:

${ }^{1}$.for the event J4OJ3 on Feb 18, observers suspected some time-recording error in the observations due to the lack of experience

2 .the event J1EJ4 on Feb 28 was interrupted during observing soon after the maximum drop since the eclipsed satellite entered the shadow of Jupiter

new image-processing technique (Peng et al. 2007). To reduce our normal CCD imaging observations, the newest theory of L1 (Lainey 2004a, 2004b) in the IMCCE for the Galilean satellites and JPL DE405 for Jupiter was adopted to compute their theoretical positions. The reduction of mutual events is the same as that in Emelyanov \& Gilbert (2006). Specifically, we do not model the apparent relative motion of one satellite with respect to the other but the deflection of the observed relative motion from the predicted motion provided by L1 ephemeris instead. We take the reflectance properties of satellites from Hapke theory with Hapke parameters. In addition, we use the dependence of the magnitudes of Galilean satellites on angle of rotation and take the solar limb darkening into account.

\section{Results and conclusions}

After the reduction of the observations of mutual events and normal CCD imaging, we can derive the differences of observed minus computed positions $(\mathrm{O}-\mathrm{C})$ in right ascension and declination, respectively. Table 1 shows the detailed results. Despite our lack of experience for the first mutual event observation (J4OJ3) and the unexpected situation took place (J1EJ4) in our observations, it is shown that there is very good agreement for the event J1O J2 taken on Feb 20 and Feb 27, 2003. This agreement exists not only for internal observations (within $\sim 15$ mas) of the same observational type but also in the external ones (within $\sim 20$ mas) for two different types of observations. However, more observations are needed to test that. It is well known that the principal drawback of mutual event observations is the rarity of such phenomena. But for small-separation satellite-pair observations, they have obvious significance that the 50 arcsec separations occur more frequently than the mutual events - averaging about 50 per month (Pascu 1994). Therefore, we might draw a conclusion that normal CCD imaging observations for a small-separation satellite-pair may have a good precision comparable with that from the mutual event observations.

\section{References}

Arlot, J. -E. 2002, A\&AA, 383, 719-723

Emelyanov, N. V. \& Gilbert, R. 2006, A\& $A, 453,1141-1149$

Lainey, V., Duriez, L., \& Vienne, A. 2004a, A\& A, 420, 1171-1183

Lainey, V., Arlot, J. -E., \& Vienne, A. 2004b, A\&A, 427, 371-376

Pascu, D., 1994, in: L. V. Morrison \& G. F. Gilmore (eds.), Galactic and Solar System Optical Astrometry(Cambridge),p. 304-311

Peng, Q. Y., Vienne, A., Lainey, V., \& Noyelles, B. 2007, PESS (in press) 